\title{
An Assessment of an Educational Intervention Program Based on the Health Belief Model for Safer Sex Among Female Sex Workers in Selected Districts of Northern Region of Malawi
}

Donatien Twizelimana ( $\nabla$ dr.donatientwizelimana@yahoo.com )

Kamuzu University of Health Sciences

Adamson Sinjani Muula

Kamuzu University of Health Sciences

\section{Research Article}

Keywords: Toolkit, FSWs, pregnancy, contraceptives, STIs, educational intervention

Posted Date: December 20th, 2021

DOI: https://doi.org/10.21203/rs.3.rs-1000314/v1

License: (c) (1) This work is licensed under a Creative Commons Attribution 4.0 International License.

Read Full License 


\section{Abstract}

Background:

Pregnant women are at risk of pregnancy if they have unprotected sex, do not use or poorly use contraceptives in the context of penile-vaginal sex. We therefore developed an educational toolkit based on the Health Belief Model (HBM) to assist FSWs to make informed sexual and reproductive decision for safer sex before and/or after heterosexual encounter with their clients. We evaluated the educational intervention programme among FSWs and other stakeholders (nurses and clinicians).

Methods:

This was a qualitative operational research. We developed an educational tool kit based on the HBM to aid FSWs to make informed decision for safer sex behaviour. We conducted 10 in-depth interviews (IDIs) to identify the components of the educational tool kit, $5 \mathrm{IDIs}$ for modification and refining the tool, and consequently two Focus Group Discussions (FGDs) for consensus building. This process was done in Mzimba North. After the intervention we conducted 6 FGDs with FSWs and 10 IDIs with nurses and clinicians to evaluate the applicability and feasibility of the intervention among female sex workers (FSWs) in Mzimba North, and Nkhata Bay located in Northern region of Malawi.

Results:

We observed mixed opinions on the components of the educational toolkit. Female sex workers were eager to understand misinformation and misconceptions on contraceptives, right to justice, effectiveness of contraceptives. Female sex workers requested the inclusion of STIs and HIV prevention and economic empowerment in the toolkit. Overall the toolkit and the educational intervention were relevant, feasible, and applicable among the study participants.

Conclusion:

While several strategies can be used to facilitate the implementation of the evidence based intervention to improve health, our educational intervention program based on the Health Belief Model for safer sex behaviour among female sex workers was found to be feasible and applicable in our study settings. We believe that the intervention may be helpful to address sexual and reproductive health challenges encountered by FSWs in the study sites and elsewhere.

\section{Background}

Female sex workers have a disproportionate burden of HIV, they experience high risks of pregnancy, violence, sexually transmitted infections, and other adverse health outcomes [1]. Female sex workers are at 13 times higher risk of HIV acquisition than other women of reproductive age [2]. HIV is acquired from male clients or regular partners which is then transmitted to further partners including clients of sex work $[3,4]$. Further, high prevalence of unintended and teenage pregnancy has been documented among FSWs 
in several studies elsewhere $[1,4,5]$.Teenage pregnancy, on the other hand, is associated with sexually transmitted infections (STIs) including HIV, high risks of eclampsia, puerperal endometriosis, premature birth, unsafe abortions, and post-abortal infections [4,5]. Pregnant adolescents living with HIV have poor access and uptake to prevention of mother to child transmission (PMTCT) services compared to their non-adolescent pregnant counterparts [6]. There are many individual, relational, cultural, and organizational factors that are associated with FSWs' decision to have or not have children $[1,7]$. Our previous study findings in semi urban Blantyre in Malawi, suggest that female sex workers experience pregnancy under diverse circumstances [1,7] which include: inconsistent condom use, desire to be respected as mothers, request from a steady partner to have children, unmet contraceptive needs, condom failure, and low health literacy $[1,7,8]$. Given the significant burden of HIV among FSWs and intersecting risks of unintended and teenage pregnancy in this population, there is a need to assist FSWs to make informed sexual and reproductive decision for safer sex before and/or after heterosexual encounter with their clients.

Based on the above understanding we developed and used an educational toolkit to help FSWs to overcome these challenges. In this study contexts a toolkit is referred to a document that provides information or guidance. It can be educational material, timelines, and agenda template and assessment tool. Toolkits to support evidence-based interventions commonly target healthcare providers [9]. There is evidence suggesting that the use of toolkit to support the implementation of healthcare related intervention is associated with improved patient outcomes [9]. During the toolkit development female sex workers, nurses and clinicians' opinions were observed and assessed. Local misunderstandings and beliefs on contraceptives were addressed during the training.

Our intervention was guided by the Health Belief Model (Refer to figure 1). It is a theoretical model that has been widely used to explore various health related behaviours such as sexual risk behaviours, transmission of STIs including HIV, and prevention of unintended pregnancies. It is constructed from six domains: perceived susceptibility, perceived severity, perceived barriers, perceived benefit, cue to action and perceived self-efficacy $[10,11,12,13]$. Based on the concept of HBM in our study context, FSWs must gain knowledge of unintended pregnancy, STIs including HIV. They must perceive themselves as susceptible to getting unintended pregnancy or be infected with HIV and they must be convinced that getting unintended pregnancy or STIs (including HIV) is serious issue that have negative social, economic and health implications $[10,11,12,13]$. Additionally, FSWs must be convinced that it is possible to obtain control over the social, economic and personal barriers and that the barriers do not outweigh the benefits of pregnancy and STI prevention (Refer to figure 1). Likewise, an internal or external stimulus, cues to action must prompt the health behaviour of FSWs towards preventing unintended pregnancy and STI including HIV. Lastly, FSWs must believe that they are capable to avoid unintended pregnancy and or prevent STI (self-efficacy) $[13,14,15]$.

Information about adverse health outcomes related to the previous study findings were presented during the education process (perceived susceptibility and severity), and FSWs were helped to explore their 
values and attitudes and make their own informed sexual and reproductive decision and strategies (cues to action) for safe sex work $[14,15,16]$.

We sought to assess the applicability and feasibility of an educational intervention program on informed decision making for safer sex behaviour based on the Health Belief Model amongst female sex workers in Northern region, Malawi. Data on the impact of an educational intervention program based on HBM for safer sex behaviour among FSWs in Mzimba North, and Nkhata Bay districts of Northern region of Malawi are difficult to find. Hence female sex workers are at significant high risk for HIV and other STIs. They also experience a high burden of unintended pregnancies resulting from high risk sex behaviour. In order to address this gap in the literature we conducted a comprehensive educational intervention based on the Health Belief Model for safer sex among female sex workers in selected districts of Northern Region of Malawi. We developed and used a toolkit with the potential to increase their knowledge, enhance their attitude and safer sex behaviour towards pregnancy and STI prevention. We believe that the intervention may be helpful to address sexual and reproductive health challenges encountered by FSWs in the study sites and elsewhere.

\section{Methods}

\section{Study design and population}

The study was conducted between May and July 2021. This was a qualitative operational research in which we developed an educational tool kit based on the HBM to aid FSWs to make informed decision for safer sex behaviour. The study was conducted in two purposively selected districts in Northern Malawi, i.e. Mzimba North and Nkhata Bay. In Mzimba North the study was done in Mzuzu, the only city in Northern region and the third largest city in the country. It is along the main road connecting Tanzania and Malawi. It is a gate away to Lake Malawi and Nkhata Bay district. Mzuzu has many small to medium sized businesses which provide various services. It has many entertainment places, bars, hotels, and lodges. Nkhata Bay borders Mzuzu. In these study sites commercial sex takes place in many settings including luxury hotels, small guesthouses, roadside stops of trucks and various entertainment establishments such as bars and beaches along Lake Malawi. [17]

The study participants aged between 18 and 35 years who agreed to have exchanged sex for money or goods and consented for the study were eligible for the study participation. Female sex workers who were sick, participating in another study or coming from outside the study site were excluded. The first female sex workers were reached through Mzimba North District Health and Social Services (DHSS) offices. The office linked the research team to FSWs' chairperson who identified FSWs who were used as seeds. The later connected us to their friends in the snowball sampling process that was used.

In each of the locations, data collection was done by three research assistants. One was taking notes, the other one was facilitating the discussions, and the remaining was audio recording the discussions. The 
sample size in these two study sites was reached by saturation and redundancy. In total 40 FSWs and 15 stakeholders (clinicians and nurses) were recruited.

The first step was to identify the components of potential educational toolkit comprising elements of contraceptive methods and informed decision making for safer sex behaviour. For this process we conducted 10 IDIs. After analysing the data, we developed the draft of the toolkit based on the findings of our first objective. We also conducted 5 IDIs for modification and refining the tool kit, then two FGDs for consensus building. This process was done in Mzimba North. When the toolkit was complete, we conducted educational intervention among FSWs in Mzimba North and Nkhata bay on contraceptives, decision making on safe sex behaviour. We conducted pre and post intervention tests. We conducted 6 FGDs to evaluate the applicability and feasibility of the intervention immediately after the training (for female sex workers). After sharing the toolkit with other stakeholders (nurses and clinicians) we conducted 10 IDIs to evaluate the potential applicability (transferability) and feasibility of the contents of the toolkit.

\section{Data analysis}

Data were analysed manually using thematic content analysis relating to the objectives of the study. After data transcription, themes were identified and codes were developed. Similar themes were categorized accordingly [7].

\section{Ethical consideration}

We followed international and national ethical standards. The study was reviewed and approved by the COMREC (College of Medicine Research and Ethics Committee) of the Kamuzu University of Health Sciences-formerly College of Medicine and Kamuzu College of Nursing of the University of Malawi (certificate number P.03/21/3290 dated 02 June 2021). The Directorates of Health and Social Services of Mzimba North and Nkhata Bay granted permission to conduct the study. All participants provided written informed consent. Cash reimbursement of transport, time and inconvenience of Malawi Kwacha (MK) 9000.00 (approximatively10 United States Dollars at the time of data collection) was given to all study participants.

\section{Results}

\section{Female sex workers' characteristics}

We recruited 40 study participants. The sample had a mixture of brothel and street based FSWs. Twentyeight had less than 20 years. Thirty-four FSWs had completed primary school education as their highest level of education. 
Table 1

Socio-demographic characteristics of female sex workers $(\mathrm{N}=40)$.

\begin{tabular}{|lcc|}
\hline Characteristics & N & $\%$ \\
\hline Age $(\mathbf{N}=40)$ & & \\
\hline Less than 20 years & 28 & 70 \\
\hline $20-30$ years & 2 & 5 \\
\hline More than 30 years & & \\
\hline Education & 34 & 85 \\
\hline Primary & 6 & 15 \\
\hline Secondary & 0 & 0 \\
\hline Tertiary & & \\
\hline Place of birth & 29 & 72 \\
\hline Rural & 11 & 27 \\
\hline Urban & & \\
\hline Other characteristics & 21 & 52 \\
\hline Consistent condom use & 33 & 82 \\
\hline STls & 36 & 90 \\
\hline FSWs desired to have few children & 32 & 80 \\
\hline Fear of side effects of contraceptives & 28 & 70 \\
\hline Unmet contraceptive needs & & \\
\hline
\end{tabular}

\section{Identification of the components of the toolkit}

Different themes emerged among the study participants:

\section{Misinformation and misconceptions on contraceptives}

The study participants highlighted the needs to clarify some misconceptions on contraceptives when teaching FSWs on safer sex behaviour and contraceptives. Twenty-one FSWs narrated that indeed there are myths and misconceptions among FSWs and the general population. 
Many of us believe that once women start taking the contraceptives, it is very unlikely that she will conceive again [Age range 20-25]

Woman always have watery vaginal discharges while on contraceptives. Most of our clients dislike them. [Age range 20-25]

Contraceptives reduce libido both in males and females. "Explain in details. Asked the data collector" Males also are affected by the contraceptives because they always have sex with their partners who are on contraceptives. [Age range 25-30]

Some women believe that after unprotected sexual intercourse douching or urination works as contraception method [Age range 15-20]

\section{Inclusion of STI and HIV prevention measures}

Study participants emphasised that the training should also focus on prevention measures against STI and HIV.

You know these women, they sleep with so many men and most of the time they don't use condoms. If you don't teach them how to protect themselves properly, all of them will be infected with STIs including HIV. Therefore it is very important to gain knowledge on how to protect ourselves [Age range 30-35]

Despite using condoms people are still getting infected with HIV and STIs. Is it possible to find out the reasons why it is happening like that? [Age range 30-35]

The use of PREP and PEP should be well explained to FSWs. Only very few FSWs are aware of them. [Age range20-25]

\section{Effectiveness of contraceptives}

Female sex workers narrated their experience while and after using the contraceptives. They have negative perceptions resulting from the side effects. Some proposed to make good and better contraceptives because the current ones affect their business negatively. During our intervention there was explanation of contraceptives and different alternatives.

Contraceptives prevent pregnancy... this is good. But they cause bleeding, and loss of libido. We are FSWs, how can you approach a client when you know that you are bleeding? [Age range 25-30]

Taking the pills on daily basis is not helpful. You may forget! But if you find us pills which can work for long time, we can appreciate. Injections make us bleed continuously. [Age range 30-35] 


\section{Female sex workers' empowerment}

Female sex workers explained the needs for their empowerment. Without alternative reliable sources of income FSWs have only one option of survival, sex work.

It's not our wish to be FSWs. This is the result of poverty. Find us the source of income then we will forget the sex work. Teach us other ways to generate money, we are tired of sex work. [Age range 20-25]

Please link us to organisations which can assist us. We want to do business. We can pay back after sometimes. You can't became rich as result of sex work. [Age range 25-30]

\section{Right to justice}

Female sex workers expressed the need to have a legal framework allowing them the to operate freely. Currently whenever they complain about brutality and many forms of harassment from several groups' perpetrators, they are rarely heard by the law enforcers.

In many circumstances the police officers don't listen to us, even when the issues presented to them are genuine. We are not protected. Please talk to the police to listen and understand our concerns. We are like any other citizens of this country. [Age range 30-35]

In other country FSWs are much protected. Here we are beaten anyhow, raped, and abused. Please protect us. [Age range 25-30]

\section{Access to contraceptives and other health care services}

Female sex workers expressed their concerns about the availability of contraceptives and other health care services (ART, PEP, PrEP, screening and counselling services). This problem is very common in health centres.

It's good that FSWs will be conversant with contraceptives but although the Government provides the contraceptives for free of charge, most of the time they are out of stock in many health centres [Age range 25-30]

Many organisations assist FSWs with condoms, lubricants. They come to test us while in the field. We will appreciate if health care providers bring the contraceptives to our homes or in the field. (Age range 2530)

\section{Evaluation of the toolkit}


Qualitative method was used to evaluate the toolkit and the intervention. Feedback from FSWs, healthcare providers (Nurses and clinicians) on the relevance, content, structure, applicability and feasibility was sought.

\section{Relevance of the toolkit}

Regarding the overall evaluation of the toolkit, the majority $(70 \%)$ reported it was relevant, satisfactory and useful. It was perceived to have potential to positively impact behaviour change among FSWs.

I am satisfied with the content of the toolkit. I managed to learn a lot during the training, the facilitator explained the content of the toolkit very well. Issues related to myths and misconceptions were clearly addressed. We have an idea of the type of contraceptive suitable for us. We will start using dual method as soon as possible. We acquired adequate information to prevent STI and unintended pregnancy. [Age range 20-25]

This document has reminded me what we have to do as nurses and clinicians. We must look after them (FSWs). We should not be judgemental. [Clinician. Age range 25-30]

This document is very useful and relevant to all of us: FSWs, health workers, and the community. If we don't protect FSWs against diseases, they will get sick and during sex work they may meet our husbands, children and/or our relatives. Think about what will happen if your husband gets HIV from FSWs, you will be infected as well. Let us use the toolkit for our protection. [Nurse. Age range 30-35]

I think the best way to be heard by authorities, we must organise in groups or join other groups then we present our issues as a group. Otherwise we will not have the right to justice. This discussion was very important because now we have some ideas on issues related to right to justice. [FGD 1]

\section{Organization and presentation of the toolkit}

Sixty per cent (60\%) of the FSWs reported that the toolkit is very well organized and presented. Fifty-five per cent of the clinicians said although the toolkit organisation was good some slides were very long. There was need for some modification.

The document is well presented. But it can be better if it is produced in the form of book (hard copy) so that people without computers (power point facility) can access and use it as well [FGD 1]

The document is well organized and presented. The facilitator explained using simple language to accommodate everybody. It will be better if it is translated in different vernacular languages spoken here in Malawi [FGD 1]

The document is well presented and organized. All the challenges encountered during sex work were included and possible solutions were provided. [FGD 3] 


\section{Applicability of the toolkit and the intervention}

The training was made easy by this toolkit, we managed to learn a lot. We can now easily transfer the knowledge gained to other fellow female sex workers and friends. [FGD 2]

We will be very confident to explain or teach FSWs on contraceptives and safe sex behaviour using the same toolkit. We will appreciate if you share with us the slides so that we can use them elsewhere during teaching sessions. [FGD 3]

This intervention was very important. It brought FSWs closer to the health care providers. We will not be judgemental and FSWs will not be afraid to approach us whenever there is a need. I have shared already the toolkit with my fellow health care providers. [FGD 4]

\section{Feasibility of the intervention}

The intervention was conveniently done. Due to this intervention, there is now closeness between FSWs and health care providers. This rarely happens. [Age range 25-30]

I liked the learning atmosphere. FSWs were very open. They expressed their views and they were eager to learn. The facilitator was very accommodative. [Clinician. Age range 30-35]

\section{Discussion}

In this study we identified the components of a potential educational toolkit comprising elements of contraceptive methods and informed decision making for safer sex behaviour. We also evaluated the applicability and feasibility of the toolkit and the educational intervention program in two selected districts of Northern Malawi.

Our study participants were young, with the majority (70\%) being less 20 years (Refer to table 1). Myths and misconceptions were very prevalent among these teenage FSWs. This is consistent with findings from studies done elsewhere [18].

Female sex workers may recognize the importance of dual protection for HIV/STI and pregnancy prevention but myths and misconceptions, such as fear for being tested for HIV at the family planning clinic, concerns about watery vaginal discharge, feared infertility from contraceptives use all act in combination to interfere with the uptake of contraceptives [18]. Adolescent pregnancies are a public health problem that occurs in both developed and developing countries. Around the world adolescents' pregnancies are more likely to occur in marginalized communities such as female sex workers, they are associated with myths and misconceptions, poverty, lack of education, and unmet contraceptive needs. Complications during pregnancy and childbirth are the leading causes of death for 15 to 19 years globally [19]. Demystification through education intervention is one of the potential approach to address teenage pregnancy. The study evaluation revealed that many FSWs were satisfied with the 
demystification process, misconceptions were cleared in the study group. Therefore we suggest that the approach can be extrapolated in other settings.

We included prevention of STI and HIV in the toolkit upon request by stakeholders (FSWs, nurses and clinicians). As indicated earlier STIs including HIV are prevalent among FSWs. In Malawi HIV prevalence was highest among Female Sex Workers at $49.9 \%$ followed by Men who have Sex with Men at $12.8 \%$ and lastly clients of female sex workers at $12.1 \%$ [20]. Logically and according to study findings individual's knowledge about a disease or health condition positively affects their attitude towards the diseases prevention and my reduce risk-taking behaviour $[21,22,23]$. However despite the knowledge of STI and HIV prevention some FSWs fail to negotiate for condom use due to financial constraints. Money driven sex was reported in many study settings $[24,25,26]$. This is one of the contributing factors of high prevalence of HIV among FSWs. Our study findings suggest that FSWs are aware of this problem. This can be explained by their request to included FSWs empowerment in the educational toolkit. Female sex workers requested to be linked to organizations which can assist them to achieve financial independency. They seem to be desperate and want to come out of sex work. They reported that nobody became rich through prostitution. This highlights the needs for economic empowerment of female sex workers. In Indian intervention aimed at empowering FSWs was successful and prevented many FSWs from many adverse health outcomes [27]. We strongly recommend that such programmes can be used to assist FSWs in our settings and elsewhere. In our education toolkit FSWs were advised to associate with other FSWs in Malawi through National FSWs association of Malawi where issues related to economic empowerment of FSWs are discussed and addressed. Many FSWs were not aware of this organisation. They appreciated our educational intervention which is an` eye opener to the study participants and other FSWs in other settings.

Sex work is not explicitly legalised in Malawi. Report suggests that female sex workers are abused and harassed, by the police and male clients [20]. It is also reported that no actions were taken after FSWs reported cases of rape, violence to the police officers [20]. Based on this background and the current situation of FSWs in Malawi, we assume that FSWs requested the topic of right to justice to be included in the educational toolkit. During the training process, we shared with FSWs the study findings on criminalization of sex works. There is no evidence that criminalizing sex work deters those who may sell or buy sex. Instead, the evidence shows that criminalization, whether full or partial (the latter only targets buyers) makes sex work more dangerous, drives sex workers into more isolated locations, impedes the use of safety and harm reduction strategies, makes it more risky to report violence and abuse from clients, managers, and law enforcement, and increases risk of exposure to HIV and other STIs $[28,29]$ .Female sex workers highlighted the need to be considered like any other human being when they are seeking legal services. Female sex workers are key population, they have negative impact on public health, therefore actions such decriminalization of sex work may be used as one of the risk reduction intervention for FSWs. There are few countries worldwide that legalised sex work. In Senegal sex work was legalised since 1969. A study done on the effect of becoming registered FSWs on physical health and well-being revealed that in Senegal, becoming a registered FSW leads to a greater use of HIV/STI prevention services and reduced STI prevalence. [30] 
In Malawi, according to MDHS $2015-2016$ overall, $68 \%$ of all women currently using modern contraceptives were informed at the time they started the current episode of method use about the side effects of the method, what to do if they experience side effects, and other available methods [31]. While data on FSWs is missing in the report, findings in our previous study suggested that more than half (57\%) of FSWs had poor knowledge of contraceptives [32]. We therefore focused on contraceptives' knowledge, side effects, and other alternatives during FSWs training. Female sex workers were encouraged to think about and identify their contraceptives needs. Our intervention provided basic information on a range of options to help FSWs identify possible methods for prevention of pregnancy, HIV, and other STIs. The tool kit provided also information about where they can obtain contraceptive services. Eighty five percent of FSWs interviewed were satisfied with the training intervention and the content as well as explanations in the tool kit.

The toolkit used during the educational intervention was supported by the research evidence. The information regarding the evaluation of the toolkit either as a whole or for its components was collected after the intervention. The evaluation aimed at assessing changes in practice and knowledge as a result of the intervention and the toolkit use requires further studies. We would have extended the evaluation over a period of time in order to assess behaviour change. The Stages of Change model shows that for most persons a change in behaviour occurs gradually, with the patient moving from being uninterested, unaware or unwilling to make a change (precontemplation), to considering a change (contemplation), to deciding and preparing to make a change. Genuine, determined action is then taken and, over time, attempts to maintain the new behaviour occurs [33]. We used Health Belief Model to assist FSWs to overcome this challenge. Female sex workers perceived that they are susceptible to unintended pregnancy and its complications, HIV and other STI transmission. Since they did not underrate the problem (perceived severity) we can anticipated the likelihood of contraceptive use, safer sex behaviour. This is consistent with the data collected. The majority $90 \%$ of the study participants reported that the toolkit is helpful and relevant. They reported that "we will start using dual method as soon as possible". After demystification we also believe that FSWs will change behaviour in the near future.

The ultimate objective of the research is to integrate the research findings into practice to inform and improve health. Proper implementation of research findings also known as knowledge translation has been used to increase awareness and improve health. This process can be done through printed educational materials, (e.g. audio-visual materials, electronic publications), meetings, educational outreach [34]. In our intervention we used a multifaceted approach tailored with FSWs with targeted messages (contraceptives, and safer sex behaviour), and attention to identified barriers to change (myths and misconceptions). This approach made our intervention feasible. The majority of study participants reported that the intervention will positively impact the closeness of FSWs and the health care providers. This implies that FSWs may access easily health care services including the uptake of contraceptives. [35]

We shared the toolkit with nurses and clinicians. After 4 weeks we collected information on the feedback of the toolkit. They reported to have shared the toolkit with their colleagues. It was also recommended 
that the toolkit should be in the form of a small book and the content should be translated in vernacular language. During the data collection process, FSWs also reported to have the toolkit in their respective languages. This reflects that the toolkit and the intervention are applicable in other settings but the language of the targeted community should be taken into consideration.

\section{Study limitations}

Sex work is a sensitive issue; as such there is potential for social desirability bias which in turn underestimates the magnitude of studied issue in FSWs community. There is also a possibility of recall bias and misreporting of personal experiences

\section{Conclusion}

While several strategies can be used to facilitate the implementation of the evidence based intervention to improve health, our educational intervention program based on the Health Belief Model for safer sex behaviour among female sex workers was found to be feasible and applicable in our study settings. We believe that the intervention may be helpful to address sexual and reproductive health challenges encountered by FSWs in the study sites and elsewhere.

We therefore recommend that the intervention can be scaled up to other contexts outside the study site. The toolkit used during the intervention was well organised and presented but needs modifications such as translation in local language to make it more accessible and understandable by all the users in selected districts of Northern Malawi and elsewhere.

\section{Abbreviations}

ACEPHEM Africa Centre of Excellence in Public Health and Herbal Medicine

ART Antiretroviral Therapy

COMREC College of Medicine Research and Ethics Committee

FGDs Focus Group Discussions

FSWs Female Sex workers

HBM Health Belief Model

IDIs In-depth interviews

NGO Non-Governmental Organization

MDHS Malawi Demographic and Health Survey

Page 13/18 
PMTCT Prevention of Mother To Child Transmission of HIV

PrEP Pre-exposure prophylaxis for HIV

PEP Post-exposure prophylaxis for HIV

SRH Sexual and Reproductive Health

STI Sexual Transmitted Infection

\section{Declarations}

\section{Acknowledgements:}

We acknowledge the tremendous work done by the data collection study team. We thank the research participants in all study sites in Mzimba-North.

\section{Funding:}

The study was funded by ACEPHEM through Kamuzu University of Health Sciences-formerly College of Medicine and Kamuzu College of Nursing of the University College of Medicine.

\section{Availability of data and material:}

The data sets used and/or analyzed during this study are available from the corresponding author on reasonable request.

\section{Competing interests:}

None of the authors have conflicting interests

\section{Author's contribution:}

DT contributed to the study design, data collection, data analysis, and in the writing of the report. AM contributed in the study design, interpretation of the data, writing the report and provided important intellectual content to the study process. All the authors read and approved the manuscript.

\section{Consent for Publication:}

Not applicable 


\section{Ethical approval and consent to participate in the study:}

The study was reviewed and approved by the COMREC (College of Medicine Research and Ethics Committee) of the Kamuzu University of Health Sciences-formerly College of Medicine and Kamuzu College of Nursing of the University of Malawi (certificate number P.03/21/3290 dated 02 June 2021). The Directorates of Health and Social Services of Mzimba North and Nkhata Bay granted permission to conduct the study. All participants provided written informed consent.

\section{References}

1. Twizelimana, D., Muula, A.S. Correlates of pregnancy among Female Sex Workers (FSWs) in semi urban Blantyre, Malawi. BMC Pregnancy Childbirth 20, 337 (2020).

2. Baral SD., Beyrer C., Muessig K., Poteat T., Wirtz AL., Decker MR. Burden of HIV among female sex workers in low-income and middle-income countries: a systematic review and meta-analysis. Lancet Infect Dis. 2012; 12:538-49

3. Rojanapithayakorn W. The $100 \%$ condom use programme in Asia. Reproductive Health Matters. 2006; 14: 41-52.

4. Schwartz SR., Baral S. Fertility-related research needs among women at the margins. Reprod Health Matters. 2015 May; 23(45):30-46.

5. Yakubu I., Salisu WJ. Determinants of adolescent pregnancy in sub-Saharan Africa: a systematic review. Reprod Health. 2018; 15:15.

6. Christofides NJ., Jewkes RK. Dunkle KL. Nduna M., Shai NJ., Sterk C. Early dolescent pregnancy increases risk of incident HIV infection in the Eastern Cape, South Africa: a longitudinal study. $J$ Int AIDS Soc. 2014; 17(1):18585.

7. Horwood C., Butler LM., Haskins L., Phakathi S., Rollins N. HIV-infected adolescent mothers and their infants: low coverage of HIV services and high risk of HIV transmission in KwaZulu-Natal, South Africa. PLoS One. 2013; 8(9): e74568.

8. Twizelimana, D., Muula, A.S. Actions taken by female sex workers (FSWs) after condom failure in semi urban Blantyre, Malawi. BMC Women's Health 20, 273 (2020).

9. Weldegebreal R., Melaku YA. Alemayehu M., Gebrehiwot TG. Unintended pregnancy among female sex workers in Mekelle city, Northern Ethiopia: a cross-sectional study. BMC Public Health. $2015 ; 15: 40$.

10. Dykes PC., Duckworth M., Cunningham S., Dubois S., Driscoll M., Feliciano Z., Ferrazzi M., Fevrin FE., Lyons S., Lindros ME., Monahan A., Paley MM., Jean-Pierre S, Scanlan M. Pilot Testing Fall TIPS (Tailoring Interventions for Patient Safety): a Patient-Centered Fall Prevention Toolkit. Jt Comm J Qual Patient Saf. 2017 Aug;43(8):403-413

11. Champion, V.L., and Skinner C.S. The Health Belief Model. Health Behaviour and Health Education; Theory, Research, and Practice. Jossey-Bass, San Francisco. 2008; 45-65. 
12. Carpenter CJ. A Meta-Analysis of the Effectiveness of Health Belief Model Variables in Predicting Behaviour. Health Communication. 2010; 25: 661-669.

13. Janz NK., Becker MH. The Health Belief Model: A Decade Later. Health Education Quarterly. 1984; 11(1):1-47

14. Glanz K., Rimer BK., Viswanath K. Health behaviour and Health education: Theory, research, and practice. 4th ed. Jossey Bass; 2008. 1-590 p.

15. Rickard W., Growney T., Occupational health and safety amongst sex workers: a pilot peer education resource, Health Education Research, Volume 16, Issue 3, June 2001, Pages 321-333,

16. Yakubu I., Garmaroudi G., Sadeghi, R.. Assessing the impact of an educational intervention program on sexual abstinence based on the health belief model amongst adolescent girls in Northern Ghana, a cluster randomised control trial. Reprod Health 16, 124 (2019).

17. Herold ES., The health belief model: can it help us to understand contraceptive use among adolescents? J Sch Health. 1983 Jan; 53(1):19-21.

18. Population and housing census Main Report 2018, Malawi National Statistical Office Retrieved on 12 September 2021

19. Ochako R., Okal J., Kimetu S. Female sex workers experiences of using contraceptive methods: a qualitative study in Kenya. BMC Women's Health 18, 105 (2018).

20. Mothiba T.M., Maputle, MS., Factors contributing to teenage pregnancy in the Capricorn district of the Limpopo Province, Curationis. 2012;35(1), Art. \#19, 5 pages.

21. Malawi Biological and Behavioural Surveillance Survey Report 2019-2020. http://www.nsomalawi.mw/images/stories/data_on_line/demography/bbss/BBSS\%2020132014\%20Report.pdf. Accessed 112021.

22. Tanaka Y., Kunii O., Hatano T., Wakai S. Knowledge, attitude, and practice (KAP) of HIV prevention and HIV infection risks among Congolese refugees in Tanzania. Health Place. 2008; 14(3):434-52.

23. Ezedinachi E., Ross M., Meremiku MM. Essien E. The impact of an intervention to change health workers'HIV/AIDS attitudes and knowledge in Nigeria: a controlled trial. BMC Public Health. 2002; 116:106-12.

24. Dong X., Sun M., Wang J., Yang Z., Hu B. Understanding the hierarchical relationships in female sex workers' social networks based on knowledge, attitude, and practice. Int $J$ Environ Res Public Health. 2019; 16(20):3841

25. Steiner M., Piedrahita C., Glover L., Joanis C. Can condom users likely to experience condom failure be identified? Fam Plan Perspect. 2014; 25(5):220-6.

26. Wong ML., Lubek I., Dy BC., Pen S., Kros S. Social and behavioural factors associated with condom use among direct sex workers in Cambodia. Sex Transm Infect. 2003; 79:163-5.

27. Choi SY., Holroyd E. The influence of power, poverty and agency in the negotiation of condom use for female sex workers in mainland China. Cult Health Sex. 2007; 9:489-503. 
28. Swendeman D., Basu I., Das S., Jana S., Rotheram-Borus MJ. Empowering sex workers in India to reduce vulnerability to HIV and sexually transmitted diseases. Soc Sci Med. 2009; 69(8):1157-1166.

29. Moore KE., Stuewig JB., Tangney JP. The effect of stigma on criminal offenders' functioning: a longitudinal mediational model. Deviant Behav. 2016;37(2):196-218.

30. Herrmann, F. Building a Fair and Just New York: Decriminalize Transactional Sex. Hastings Race and Poverty Law Journal. 2018; 15(1), 65.

31. Ito S., Lépine A, Treibich C. The effect of sex work regulation on health and well-being of sex workers: Evidence from Senegal. Health Econ. 2018 Nov; 27(11):1627-1652.

32. National Statistics Office (NSO) [Malawi] and ICF International. Malawi Demographic and Health Survey 2015-2016: Key Indicators Report. Zomba, Malawi and Rockville, Maryland: NSO and ICF International; 2016.

33. Twizelimana, D., Muula, A.S. Unmet contraceptive needs among female sex workers (FSWs) in semi urban Blantyre, Malawi. Reprod Health 18, 11 (2021). https://doi.org/10.1186/s12978-020-01064-w

34. Prochaska JO., DiClemente CC., Norcross JC. In search of how people change. Am Psychol. 1992; 47:1102-4.

35. Grimshaw J, Eccles M, Hill S, Squires J. Knowledge translation of research findings. Implement Sci. 2012; 7:50

36. Ma H, Loke AY. Nurses' attitudes toward female sex workers: A qualitative study. Nursing Ethics. 2021;28(4):563-574.

\section{Figures}




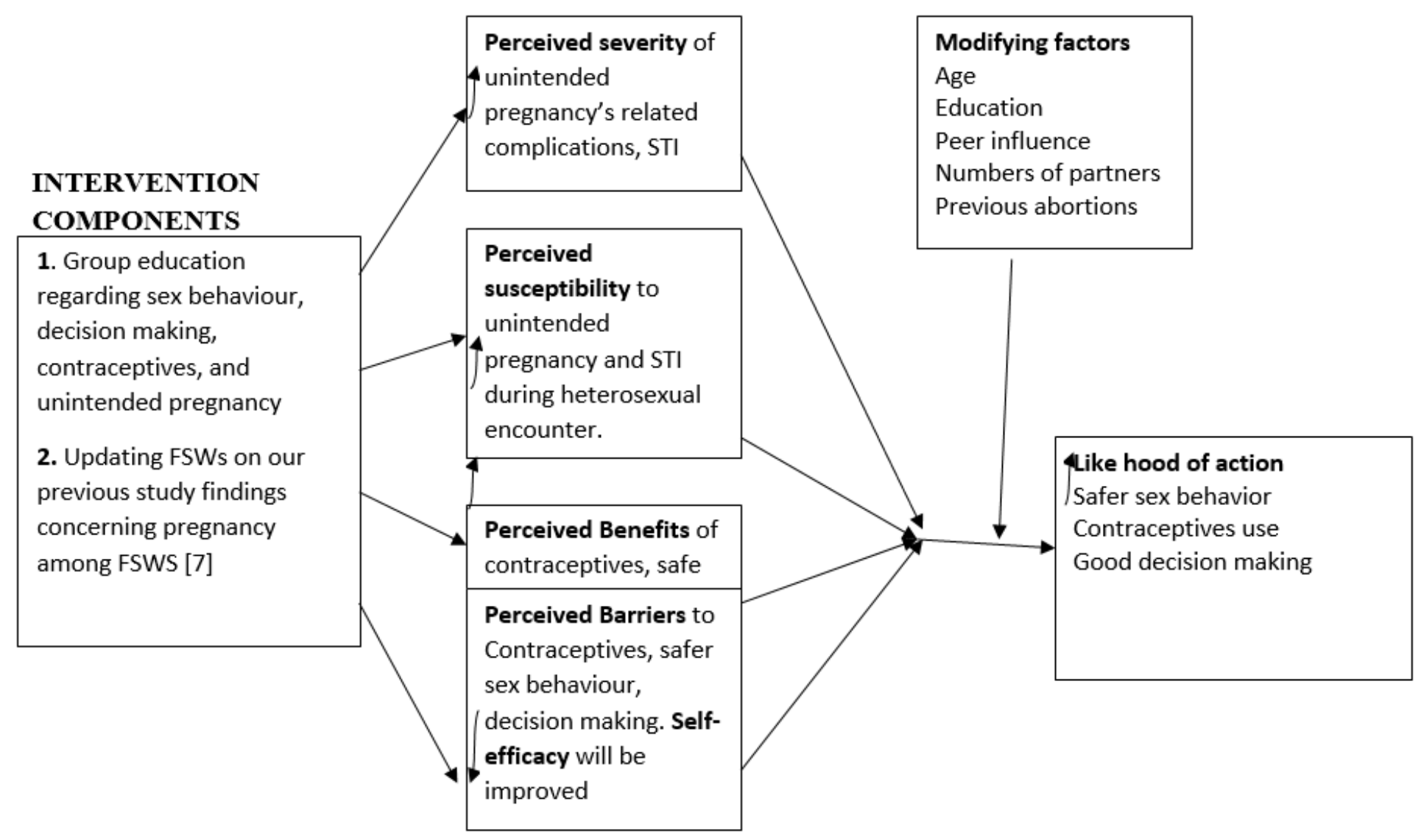

Figure 1

Study's diagrammatical representation of a Health Belief Model

Key: During the intervention FSWs were trained on safer sex behaviour, decision making, contraceptives 'use, and issues related to unintended pregnancy. They were updated on our previous study findings concerning pregnancy among FSWS [7]. After the training we anticipated increased perceived severity, perceived susceptibility, and perceived benefits. We also anticipated reduced perceived barriers and improved self-efficacy. Furthermore, they may have increased safer sex behaviour, improved contraceptives 'use and good decision making.

\section{Supplementary Files}

This is a list of supplementary files associated with this preprint. Click to download.

- BMCWHSupplementarymaterial1.docx 